\title{
Novel Approaches to Diagnose COVID-19
}

\author{
Solaf Jawhar Ali \\ Nursing Department \\ Technical College of Health \\ Sulaimani Polytechnic University \\ Sulaimani, Iraq \\ Solaf.jawhar@spu.edu.iq
}

\begin{tabular}{l} 
Article Info \\
\hline Special Issue on \\
Coronavirus (COVID-19) \\
DOI: \\
10.24017/covid.7 \\
Article history: \\
Received: 9 May 2020 \\
Accepted: 15 May 2020 \\
\hline
\end{tabular}

Keywords:

SARS-CoV-2, COVID-19, diagnosis, testing.

\begin{abstract}
An widespread of severe acute respiratory syndrome coronavirus 2 (SARS-CoV-2) in December 2019 has occurred worldwide. Public health agencies are in need of developing diagnostic tools which will have a major impact in tracking the virus and suppressing the transmission. Diagnosis of the disease is based on clinical symptoms, epidemiological history and laboratory examinations. Severe acute respiratory diseases with fever and ,cough and dyspnea, are used as the case definition to select people for testing. Different samples taken from the human body such as oropharyngeal $(\mathrm{OP})$ and nasopharyngeal $(\mathrm{NP})$ swabs are used to detect the virus. SARS-CoV-2 can be detected with different methods in the laboratory including real time RT-PCR, chest CT scan and immunoassays. Viral nucleic acid testing has played important role in control COVIDI-19 outbreak. More recently, a new CRISPR-based DETECTR assay has been developed to detect COVID-19. This test is rapid ( $30 \mathrm{~min})$, lowcost, and precise for identification of SARS-CoV-2. In addition, immunoassays and medical imaging can use as supplementary tests, combined with RT-PCR. This review is conducted to summarizes the current information on the present diagnostic approaches for SARS-CoV-2.
\end{abstract}

Copyright (0) 2020 Kurdistan Journal of Applied Research. All rights reserved.

\section{INTRODUCTION}

Coronaviruses are single-strand RNA viruses (26-32 kb), with around 40 described species that cause disease in birds and mammals. Severe acute respiratory syndrome coronavirus (SARS-CoV 2003-2004) and Middle East respiratory syndrome coronavirus (MERS-CoV 2012) have caused endemic diseases in past decades with a high case fatality rate (CFR). The severe acute respiratory syndrome coronavirus 2 (SARS-CoV-2) was first reported in December 2019 from Chin and causes COVID-19, as named by the World Health 
Organization (WHO) in February 2020. SARS-CoV-2 is similar to its coronavirus predecessors SARS (79\%) and MERS (50\%) and was declared a pandemic on March 11, 2020. According to genetic data, the COVID-19 pathogen is considered as a member of the betacoronavirus genus, and can attach to the human angiotensin converting enzyme 2 (ACE2) receptor [1]. Similar to the two previous coronaviruses that causes pandemics, SARS-CoV-2 is a zoonotic disease, transmitted to humans by infected animals, most likely bats. COVID-19 is spread from human to human by direct contact, or indirect contact with surfaces or objects used by an infected person [2]. Furthermore, the virus can survive as aerosols in the air for around three hours and cause infection in the population. There is little evidence on oral-fecal transmissibility of the virus, but in one study, COVID-19 RNA was detected in fecal specimens of patients with gastrointestinal symptoms (2-10\%) such as diarrhea. Therefore, fecal-oral transmission should be considered as a possible infection method during case tracing [3]. SARS-CoV-2 moved through many regions in China more rapidly than the previous pandemics. According to the latest data, there are more than 3.6 million confirmed cases of SARS-Cov-2, with 253,000 deaths and 1.2 million recoveries. Despite MERS-CoV and SARS-CoV having a higher case fatality ratio (CFR), SARS-CoV-2 has caused more deaths due to its larger case numbers and because it is more infectious [2]. The incubation period for COVID-19 based on WHO reports varies from 2 to 14 days in human to human transmission, with the average incubation period recorded as 5-6 days [3]. Typical symptoms of SARS-CoV-2 are fever, cough, dyspnea, viral pneumonia and lower respiratory tract disease in older patients and in any age groups who have serious underlying medical conditions. However, there are also asymptomatic laboratory-confirmed cases; therefore, it is very important to increase the number of laboratory tests carried out in order to overcome the transmission of the virus and stop the pandemic [4].

\section{METHODS AND MATERIALS}

In order to identify relevant papers in this study, a search was conducted in the different databases such as Science Direct, Pub Med, Google Scholar and Scopus. The result of the broad search was scanned by title and abstracts to retrieve relevant articles. Any articles without relevant title and/or abstract about diagnosis of COVID-19 were excluded. Subsequently, I checked for the full text of the papers. The reference lists of all studies identified by the above techniques have been checked.

\section{RESULTS}

\subsection{Diagnosis strategies for SARS-CoV-2}

A rapid diagnosis of SARS-CoV-2 is crucial in preventing transmission of the disease. Various methods have used to diagnose the virus including clinical manifestations, epidemiological history and laboratory examinations, and these will be discussed here.

\subsection{Clinical manifestations}

Severe acute respiratory diseases with a cough and shortness of breath, are used as the case definition to select people for testing. This strategy involves typical symptomatic presentation, but inadequately identifies unusual manifestations, such as cases without, or only very mild, respiratory symptoms[5]. One modeling study stated that up to $86 \%$ of patients might have been missed in China, and reports of patients with unusual presenting symptoms are increasing globally[6]. Diagnosis of the new pathogen based on clinical symptoms is difficult and preliminary disease symptoms are usually nonspecific. Mild common cold symptoms including sore throat, dry cough, low fever or body aches are prevalent. If the clinical manifestations worsen after a few days, people usually visit emergency units. Due to the wide range of clinical symptoms, research on biomarkers and clinical criteria predicting prognosis is of first priority to facilitate differentiation of mild cases needing limited monitoring and more severe cases that may require more interventions in infection's early stages.

\subsection{Nucleic acid detection technology}


Viral nucleic acid testing has played significant role in control of COVID-19 outbreak. Realtime reverse transcription polymerase chain reaction (RT-PCR) is used in routine confirmation of cases of SARS-CoV-2. The specificity and sensitivity of RT-PCR is dependent upon preanalytical and analytical factors such as the sample type, timing of sample collection, number, shipment and packaging of the samples which might affect the diagnostic accuracy of the test. Clinical samples could be collected from saliva, nasopharyngeal (NP), pharyngeal (OP), blood and stool. At the early stage of infection, both NP and OP are recommended. However, in China, SARS-CoV-2 RNA extraction is mostly via NP swabs rather than OP swabs. At the later stage of the disease, a rectal swab can be used for RT-PCR, as a high viral load was demonstrated in the stool of patients[7].

Target selection for RT-PCR is one of the factors that have a role in accuracy detection of SARS-CoV-2. Many gene targets that are needed for viral replication have used to specify the virus by PCR assay such as the transmembrane region (M), envelope glycoprotein spike (S), envelope (E), nucleocapsid (N) and helicase (Hel) genes [8]. Different researchers have used different viral genes targets for RT-PCR assays. However, the optimization of each target in different locations is important. Moreover, using more than one target is essential to avoid any misleading as a result of unspecific reaction with other coronaviruses. In the United States, in order for a positive laboratory confirmation of infection, both tests of nucleocapsid proteins $\mathrm{N} 1$ and N2 should be positive. Three targets in the N gene can be used too[9]. Furthermore, in China two or more targets are used to detect SARS-CoV-2. Species-specific accessory genes are other possible target genes for RT-PCR that are essential for viral replication such as open reading frames ORF1a and ORF1b, hemagglutinin-esterase (HE) and RNA-dependent RNA polymerase (RdRp) assay. E gene target is first recommended by WHO to detect the virus followed by confirmation test using (RdRp) assay [8]. In Germany, RdRP, E and N genes are checked. In one study, authors compared different PCR systems and different RT-PCR assays and reported that one-step RT-PCR systems are more efficient and sensitive for diagnosis of SARS-CoV-2 [10]. Another research group from Spain has provided a simple, rapid and inexpensive method to identify patients infected by SARS-CoV-2 with high specificity and sensitivity. This protocol includes a simple step for RNA Extraction (2-propanol precipitation) followed by one-step RT-qPCR for both N1 and N2 genes [11].

Despite RT-PCR being a common genetic method for SARS-CoV-2 diagnosis, it has some limitations. First, a particular target locus in a specific region can be amplified by RT-PCR; yet, if this region is absent there will be a negative false result. Therefore, it is important to select a conserved region in each target to avoid the effect of genetic mutations in more variable regions, which might occur especially when the virus introduced in new populations. Additionally, RT-PCR is unable to provide total viral genome data, which is crucial for full development of diagnosis, treatment and prevention (vaccine) strategies of the disease. Finally, RT-PCR requires expensive instrument and efficient technicians with qualified laboratories, which may not be available to all countries worldwide. This limitation of false result RT-PCR could be overcome by deep nucleic acid sequencing such as next generation sequencing performed to define any future mutations in the viral genome. Whilst these methods are sensitive and accurate for viral diagnosis, they are time consuming and laborious. The multidisciplinary group of researchers from USA, Austria and china developed a new diagnostic technology based on whole genome sequencing (POLAR protocol). This method is reliable, inexpensive and can detect the virus even before the infected person becomes contagious. However, this technology is comparatively slow as it requires $24 \mathrm{~h}$ from sample collection to diagnostic result [12]. Due to limited availability of equipment and reagent tests to diagnose COVID-19, therefore, there is still an urgent need for a new rapid technology such as CRISPR.

\subsection{CRISPR based DETECTR assay}

Recently, a new CRISPR-based DETECTR assay has been developed to detect SARS-CoV-2 a short time ( $40 \mathrm{~min})$. This technology combined high-fidelity CRISPR detection enzyme with isothermal amplification using loop-mediated amplification (RT-LAMP) to provide fast results. After viral RNA extraction, E and N2 genes of SARS-CoV-2 are amplified using 
isothermal recombinase polymerase amplification (RPA) and subsequently Cas12a (CRISPR detection enzyme) was used to detect the specific sequence in the viral genome. To visualize the result, lateral flow strips and a biotin reporter made to catch labeled nucleic acids were used. Simple apparatus is required for this test including pipettes, tips, plastic tubes, heat blocks or water bath, reagents, nuclease-free water, and lateral flow strips. Therefore, this new CRISPR-based DETECTR assay can overcome the limitation of expensive equipment that is required for RT-PCR. This assay is very sensitive and can detect 10-100 copies of SARSCoV-2 RNA per microliter. The sensitivity and specificity of infection detection are 97 percent 100 percent, respectively. Furthermore, the same test is able to be utilized to detect SARS-CoV-2 in saliva with a high viral load without carrying out RNA extraction. The DETECTR system was validated in 2018 for detecting human papillomavirus (HPV) in human samples and accurate results were demonstrated. Developing portable lyophilized reagents and microfluid cartridges run the assay could enable the tests to be used outside laboratory settings, such as in local emergency units, health clinics, airports and other places. However, usually an RNA extraction step is still required for this assay and the FDA approval of this assay has not yet been obtained [13].

\subsection{Medical imaging}

False-negative results of RT-PCR could lead to missed diagnosis for some patients with suspected COVID-19. Therefore, medical imaging such as computed tomography (CT), positron emission tomography-CT (PET/CT), lung ultrasound, and magnetic resonance imaging (MRI) are recommended as a one of the diagnostic standards for COVID-19 and supplement examination for RT-PCR. Yan Li1 Liming Xia reported a very low rate of missed diagnosis $3.9 \%$ using the CT scan method and showed that this can be used as a standard rapid method to detect COVID-19. CT scan manifestations are used to monitor and predict different phases of COVID-19[14]. Chest CT imaging of patients infected with MERS-CoV, SARS$\mathrm{CoV}$ and SARS-CoV-2 showed ground-glass opacities and lung consolidation, which are different characteristics from other types of viruses. However, the similarity of the disease presentation of coronaviruses does mean that one of limitations of the CT method is the inability to distinguish the exact species causing the disease and therefore nucleic acid testing still the primary diagnostic tool to identify the causal agent of COVID-19 [15].

\subsection{Immunoassays}

As the lower respiratory tract is the target site for SARS-CoV-2, the test samples are largely nasal and pharyngeal swabs that are subsequently used for RNA extraction. High viral load is observed from sputum, with a positive rate from $74.4 \%$ to $88.9 \%$, and the positive rate ranging from $53.6 \%$ to $73.3 \%$ for nasal swabs. Sample collection from the lower respiratory tract requires a professional operator and a suction device. The complexity of the process might lead to false negative results. Therefore, a test which is sensitive, easy to use, fast and precise is still need to rapidly identify patients infected with SARS-CoV-2 to inhibit virus transmission[16]. In one study, an enzyme-linked immunosorbent assay (ELISA) assay for diagnosis of SARS-CoV-2 were evaluated at different times of the disease by assessing the production of $\operatorname{IgG}$ and $\operatorname{IgM}$ in serum specimens from 214 confirmed COVID-19 patients. A high positive rate was observed of the rN-based and rS-based ELISA for both IgG and IgM antibodies (80.4\% and $82.2 \%$, respectively). However the positive rate was less than $60 \%$ at the beginning of the infection for both $\operatorname{IgG}$ and $\operatorname{IgM}$, and after 35 days of infection the positive IgM dropped dramatically[17]. Similar assays are available from other research groups. For example, another group developed a rapid test where they found that IgG-IgM combined antibody tests were more sensitive and specific than separate $\operatorname{IgG}$ or IgM antibody test. The test they developed is easy to use and fast, taking around 5 minutes to get the result confirming recent infection, although not current presence of the virus. Therefore, an immunoassay can used as supplementary test combined with RT-PCR[18].

Other immunoassay tests that have been developed include an assay for identifying the nucleocapsid protein of SARS-CoV-2 using Fluorescence immunochromatographics. It is a simple, precise and fast technique for detection of COVID-19. The nucleocapsid protein of SARS-CoV-2 can be detected in the urine of the 73.6\% of detected COVID-19 patients [19]. 


\subsection{Cell culture}

For biosafety reasons, cell culture is not recommended in routine diagnostic laboratories for suspected cases. However, cell culture is a crucial test for development of vaccines and therapeutic agents. Different cell lines such as LLCMK2 are used to isolate corona virus[20].

\section{DISCUSSION}

Many factors should be considered in the choice of diagnosis method and sampling collection including viral load and infection kinetics that are influenced by host factors and the limitations of diagnostic methods with different sample types and timing. As a result of the limitations for the diagnostic methods for detecting COVID-19, there is concern that the diagnostic test for Covid-19 is not sensitive or specific enough. The problem is that tests almost never have $100 \%$ sensitivity and $100 \%$ specificity. Most of the published papers are conducted in idealized conditions, the accuracy of the results depends on the analytical validity and the diagnosis tests are not validated in community-based suspect cases. However, some diagnostic tests for COVID-19 have been agreed by the Food and Drug Administration (FDA) on February 4, 2020 based on analytical validity under an emergency use authorization without performing clinical validations[10]. Therefore, a critical next step is to design a general population sampling approach that includes accurate information about whether tested patients had clinical sequences consistent with Covid-19[21]. Both molecular and serological diagnosis methods for SARS-CoV-2 should assess their sensitivity and specificity as well as their performance across different populations. Finally, some of the studies that include in this rapid review were published when the virus was endemic, and the tests were done in asymptomatic population. Now the disease defined as a pandemic which might have effect on the diagnosis tests as a result of sampling methods and number might change in population with high probability of the disease.

\section{CONCLUSION}

- There still remains a need for a fast and specific diagnostic test to detect COVID-19

- The combined use of molecular and serological approaches is highly recommended.

- Nucleic acid testing via RT-PCR remains the gold standard of clinical analysis.

- At least two specific genomic targets are used to specify the virus by RT-PCR assay.

- The CRISPR-based assay can address the problems of lack of PCR reagents and insufficient RT-PCR machines, however this essay has not been approved yet by FDA,

- ELISA is an important supplementary technique to detect SARS-CoV-2.

- The accuracy of rapid IgM-IgG combined antibody test kit to detect SARS-CoV-2 is higher than in separated IgG or IgM antibody test.

- CT imaging is a clinical tool to monitor the progression and management of COVID19.

- Rectal swab from patients with COVID-19 pneumonia can be used for detecting SARS-CoV-2 by RT-PCR

\section{REFERENCE}

[1] L. Zhang, F. M. Shen, F. Chen, and Z. Lin, "Origin and evolution of the 2019 novel coronavirus," Clin. Infect. Dis., no. Ml, pp. 2019-2020, 2020.

[2] Q. Bi et al., "Epidemiology and transmission of COVID-19 in 391 cases and 1286 of their close contacts in Shenzhen, China: a retrospective cohort study," Lancet Infect. Dis., vol. 3099, no. 20, pp. 1-9, 2020.

[3] G. Kolifarhood et al., "19; a Narrative Review," Clin. Asp. COVID, vol. 8, no. 1, p. 41, 2020.

[4] S. S. Unhale, Q. B. Ansar, S. Sanap, S. Thakhre, and S. Wadatkar, "a Review on Corona Virus ( Covid-19 )," World J. Pharm. Life Sci., vol. 6, no. 4, pp. 109-115, 2020.

[5] P. Vetter, D. L. Vu, A. G. L'Huillier, M. Schibler, L. Kaiser, and F. Jacquerioz, "Clinical features of covid-19," $B M J$, vol. 369, no. April, pp. 1-2, 2020.

[6] R. Li et al., "Substantial undocumented infection facilitates the rapid dissemination of novel coronavirus 
(SARS-CoV2)," Science, vol. 3221, no. January, pp. 1-8, 2020.

[7] Y. W. Tang, J. E. Schmitz, D. H. Persing, and C. W. Stratton, "The Laboratory Diagnosis of COVID-19 Infection: Current Issues and Challenges," J. Clin. Microbiol., no. April, pp. 1-22, 2020.

[8] R. Lu et al., "Genomic characterisation and epidemiology of 2019 novel coronavirus: implications for virus origins and receptor binding," Lancet, vol. 395, no. 10224, pp. 565-574, 2020.

[9] M. L. Holshue et al., "First case of 2019 novel coronavirus in the United States," N. Engl. J. Med., vol. 382, no. 10, pp. 929-936, 2020.

[10] R. Konrad et al., "Rapid establishment of laboratory diagnostics for the novel coronavirus SARS-CoV-2 in Bavaria, Germany, February 2020," Eurosurveillance, vol. 25, no. 9, pp. 2-6, 2020.

[11] I.-C. A. Alcoba-Florez JGonzalez-Montelongo R, "Fast SARS-CoV-2 detection protocol based on RNA precipitation and RT-qPCR in nasopharyngeal swab samples," pp. 1-22, 2020.

[12] B. G. S. Hilaire et al., "A rapid, low cost, and highly sensitive SARS-CoV-2 diagnostic based on whole genome sequencing," bioRxiv, p. 2020.04.25.061499, 2020.

[13] J. P. Broughton et al., "CRISPR - Cas12-based detection of SARS-CoV-2," 2019.

[14] Y. Li and L. Xia, "Coronavirus Disease 2019 (COVID-19): Role of Chest CT in Diagnosis and Management," AJR. Am. J. Roentgenol., no. June, pp. 1-7, 2020, doi: 10.2214/AJR.20.22954.

[15] D. Dong et al., "The role of imaging in the detection and management of COVID - 19: a review," 2020

[16] Y. Yang et al., "Evaluating the accuracy of different respiratory specimens in the laboratory diagnosis and monitoring the viral shedding of 2019-nCoV infections," medRxiv, p. 2020.02.11.20021493, 2020.

[17] W. Liu et al., "Evaluation of Nucleocapsid and Spike Protein-based ELISAs for detecting antibodies against SARS-CoV-2," J. Clin. Microbiol., no. March, 2020.

[18] Z. Li et al., "Development and clinical application of a rapid IgM-IgG combined antibody test for SARS-CoV-2 infection diagnosis," J. Med. Virol., no. February, 2020.

[19] B. Diao et al., "Diagnosis of Acute Respiratory Syndrome Coronavirus 2 Infection by Detection of Nucleocapsid Protein," medRxiv, p. 2020.03.07.20032524, 2020

[20] M. J. Loeffelholz and Y. W. Tang, "Laboratory diagnosis of emerging human coronavirus infections-the state of the art," Emerg. Microbes Infect., vol. 9, no. 1, pp. 747-756, 2020.

[21] V. C. Bachelet, "Do we know the diagnostic properties of the tests used in COVID-19? A rapid review of recently published literature," Medwave, vol. 20, no. 03, pp. e7891-e7891, 2020. 